\title{
New advances in the neural correlates of insight: A decade in review of the insightful brain
}

\author{
SHEN WangBing ${ }^{1}$, LUO Jing $^{2,3^{*}}$, LIU Chang $^{1 *} \&$ YUAN Yuan ${ }^{1}$ \\ ${ }^{1}$ Laboratory of Cognitive Neuroscience and Department of Psychology, Nanjing Normal University, Nanjing 210097, China; \\ ${ }^{2}$ Key Laboratory of Cognition and Learning, Capital Normal University, Beijing 100083, China; \\ ${ }^{3}$ Key Laboratory of Mental Health, Chinese Academy of Sciences, Beijing 100101, China
}

Received August 7, 2012; accepted September 17, 2012; published online December 3, 2012

\begin{abstract}
The first neuroimaging study of real-time brain activity during insight problem solving was conducted almost ten years ago. Many subsequent studies have used high-resolution event-related potentials (ERPs) and event-related functional magnetic resonance imaging (fMRI) to investigate the temporal dynamics and neural correlates of insight. Recent results on the neural underpinnings of insight have led researchers to propose a neural framework referred to as the "insightful brain". This putative framework represents the neural basis of the cognitive and affective processes that are involved in insight. The insightful brain may involve numerous brain regions, including the lateral prefrontal cortex, cingulate cortex, hippocampus, superior temporal gyrus, fusiform gyrus, precuneus, cuneus, insula and cerebellum. Functional studies have demonstrated that the lateral prefrontal cortex is responsible for mental set shifting and breaking during insight problem solving. The cingulate cortex is involved in the cognitive conflict between new and old ideas and progress monitoring. The hippocampus, superior temporal gyrus and fusiform gyrus form an integrated functional network that specializes in the formation of novel and effective associations. The effective transformation of problem representations depends on a non-verbal visuospatial information-processing network that comprises the precuneus and cuneus. The insula reflects cognitive flexibility and the emotional experience that is associated with insight. The cortical control of finger movements relies on the cerebellum.
\end{abstract}

insight problem solving, insightful brain, creative thinking, neuroimaging, event-related potentials

Citation: Shen W B, Luo J, Liu C, et al. New advances in the neural correlates of insight: A decade in review of the insightful brain. Chin Sci Bull, 2013, 58: 1497-1511, doi: 10.1007/s11434-012-5565-5

The term "insight" describes an experience that is related to a state of understanding, which emerges into one's conscious awareness with sudden abruptness. Insight is the inner process that problem solvers establish as the gestalt relationship between means and goals after realizing the breadth of the problem or the manner by which the goal may be achieved from the initial state through observation. Interest in the topic of insight and insightful problem solving can be traced to early works of Gestalt psychologists [1]. Reserchers proposed the insight learning theory based on his observation of apes and defined the concept "insight" scientifically for the first time [2]. According to Köhler,

*Corresponding authors (email: luoj@psych.ac.cn; cglew@163.com) insight, as a problem-solving process, is not trial-and-error but a sudden and gestalt understanding of the problem's elements. Interest in insight has waned since the early days of the Gestalt psychologists, but a renewed interest emerged in the beginning of the 21 st century. Recent neuroimaging studies have revealed the initial neural correlates of the cognitive processes that underlie insight phenomena. Researchers have encountered numerous difficulties in the investigation of brain-based insight, but novel experimental materials and methodologies (e.g., fMRI and ERPs) have been designed to elucidate the brain mechanisms of insight. Insight plays an important and indispensable role in creative thinking, which promotes the development of human society and science and technology. Many great scientific and 
technological inventions and scientific discoveries have been solved through insight, including the delineation of Archimedes' principle and the discovery of the benzene ring structure. Many original studies on the neural basis of insight have been published in the decade following the first neuroimaging study on insight. This research on the neural underpinnings of insight has led to a neural framework that is referred to as the "insightful brain". This putative framework represents the neural basis that underlies the cognitive and affective processes of insight. The insightful brain likely involves numerous brain regions (Figure 1), including the lateral prefrontal cortex, cingulate cortex, hippocampus, superior temporal gyrus, fusiform gyrus, precuneus, cuneus, insula and cerebellum. This study reviewed cognitive neuroscience studies on insight and the roles of the different brain areas that participate in the insightful brain.

\section{Prefrontal cortex (PFC)}

The prefrontal cortex (PFC) is an important brain region in insight. The PFC primarily includes the dorsolateral prefrontal (DLPFC), ventrolateral prefrontal cortex (VLPFC), orbital frontal cortex (OFC) and the medial prefrontal cortex (MPFC). The PFC is an executive system and coordinating center of psychological activity, and this area exhibits different activation patterns during most higher psychological processes [3-6]. Insight, which is a thinking process, is undoubtedly associated with the PFC. Previous studies have shown that insight is generally linked with the DLPFC and ventral PFC [7].

Goel and Vartanian [7] adopted high spatial resolution neuroimaging techniques to investigate the neural correlates of insight that underlie matchstick problem solving. The role of the PFC in a hypothesis-generation task involving set shifts was examined in 13 normal subjects who underwent fMRI during the completion of Match Problems and baseline tasks. Subjects determined the number of possible solutions for Match Problems in each trial. Successful solutions indicate set shifts. Subjects evaluated the accuracy of hypothetical solutions to Match Problems in the baseline condition. Comparisons of Match Problems versus baseline trials revealed activation in the right VLPFC and left DLPFC. A further comparison of successfully versus unsuccessfully completed Match Problems revealed activation in the right VLPFC, left middle frontal gyrus and left frontal pole. The baseline task exhibited greater activation in the right occipital cortex than Match Problems, but this difference did not reach significance. These results suggest that the right VLPFC is a critical component of the neural mechanisms of insight problem solving. The activation in the right VLPFC that was associated with successful versus unsuccessful match problem solutions may be due to an experience of insight. However, this hypothesis is weakened by lesioning data [8], and several imaging studies [9-11] that have associated activation in right temporal lobe structures with the experience of insight did not observe activation of the right VLPFC. One neuroimaging study that focused on solution-induced insight revealed that activation in the right hippocampus [10], but not the right PFC, was associated with the experience of insight. Neuroimaging studies on creative thinking also support that the right PFC is not associated with the "Aha!" experience. These studies [12-14] revealed that the right PFC was involved in ill-structure problem solving, which suggests that the right PFC may be associated with problem presentation restructuring. Therefore, activation of the right PFC may reflect the processes of presentation restructuring or set shifting. However, these studies do not suggest that the right PFC was responsible only for the breaking of mental set and presentation restructuring. The right PFC is an indispensable and necessary functional base for the breaking of mental set and representation restructuring. Two reasons support this function. First, the breaking of mental set is one of the most important cognitive processes underlying insight, and this process could not be realized by the activation of a single brain region. Insight problem-solving tasks compared to baseline tasks activated both the right PFC and the left DLPFC. Successful insight problem-solving tasks, but not unsuccessful insight problem-solving tasks, activated the

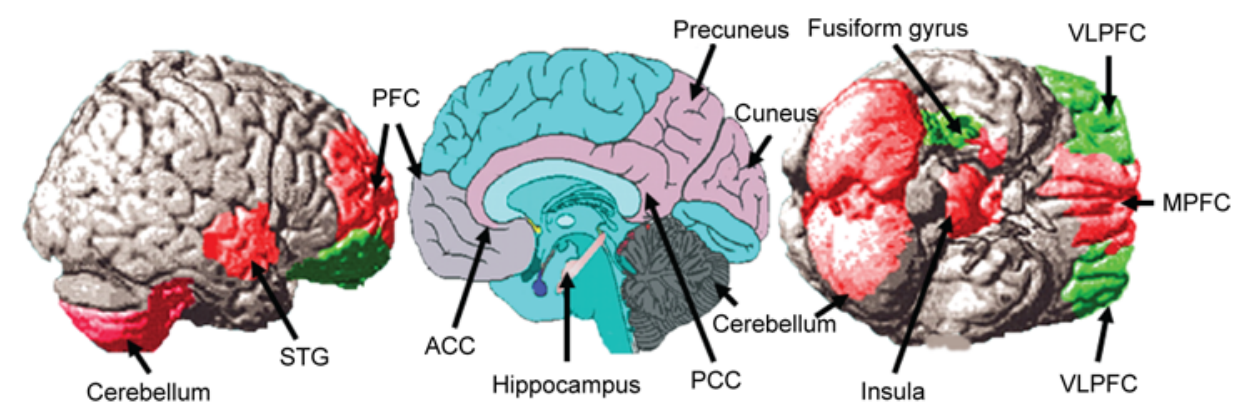

Figure 1 The neural framework of the insightful brain. The left image is a lateral view, the middle image is median sagittal section, and the right image is a bottom view. The insightful brain likely involves distributed brain regions, including the lateral prefrontal cortex (PFC), cingulate cortex (including the ACC and PCC), hippocampus, superior temporal gyrus (STG), fusiform gyrus, precuneus, cuneus, insula and cerebellum. These brain regions exhibit numerous neural connections with each other. These areas form an integrated network that is the neural basis of the cognitive and affective processes of insight. 
left middle frontal gyrus and left frontal pole [7]. Second, some studies have observed activation of the left PFC in the breaking of mental set and presentation restructuring [15].

Luo et al. [16] employed a Chinese characters chunk decomposition task to examine the role of PFC in insight problem solving. These authors asked participants to decode two Chinese characters and form two new Chinese characters. The entire process of problem solving was divided into three phases: the question phase, the hint phase and the solution phase. Two experimental conditions were used, the tight chunk decomposition (TCD) and the loose chunk decomposition (LCD). The problem in the TCD condition could be solved only if participants decomposed the character into separate strokes and moved some of the resulting strokes from the right character to the left character. Participants in the LCD condition could decompose the character into separate radicals and move one of the resulting radicals to the left character. Statistical analyses primarily focused on the LCD items that were successfully solved during the question phase and the TCD items that were solved during the hint phase. The results demonstrated that TCD in the hint presentation phase correlated with more activation in many bilateral frontal brain areas (e.g., superior frontal gyrus, middle frontal gyrus and inferior frontal gyrus) relative to LCD. The LCD items also activated these frontal brain areas. The only difference between the two types of problem solving was the degree of activation in these frontal brain areas, which suggests that the PFC participated in the TCD and LCD problem-solving tasks. Additionally, only TCD problem solving activated the right middle frontal gyrus, left inferior frontal gyrus, and two small brain areas adjacent to the right inferior frontal gyrus. The dissociation of brain activation areas that are associated with different subsequent phases is difficult due to the lower temporal resolution of fMRI. Tang et al. [17] adopted real-time ERPs to investigate the time course of insight in a Chinese chunk decomposition task because ERPs exhibit superior temporal resolution. These authors demonstrated that the unsolved problems elicited a more positive right frontal P150 during the phase of problem presentation compared to the solved problems, but the solved problems elicited a more positive left frontal P150 at the same phase compared to the unsolved problems. The unsolved problems in the hint presentation phase elicited a right frontal P2, but the solved problems elicited a left frontal P2. These results demonstrated that the problems that remained unsolved after the hint phase did not elicit a right frontal P2 compared to the problems that were solved with the help of hints, which suggests that the right frontal brain did not process problem difficulty. These data suggest that right frontal brain was prominently activated in the TCD problems and activated in the LCD problems, which also activated the PFC bilaterally. This activation might reflect the processing of tightness or problem presentation. However, the brain regions that were activated only in the TCD problems, such as the right frontal middle gyrus, right inferior frontal gyrus and the left inferior frontal gyrus, may primarily reflect the breaking of mental set and set shift.

Studies using a moderate difficult insight problem-solving task revealed that insight events activated the right inferior frontal gyrus, right frontal middle gyrus and left inferior frontal gyrus compared to non-insight events [18]. However, the right PFC exhibited significant activation in the insight condition but not the non-insight condition, which suggests the participation of the right PFC in insight. One study divided the time course of problem solving into three stages and employed a more detailed analysis of the activation areas during the early and late stages to determine the role of the right PFC in insight. Only the right ventral PFC exhibited significant activation in the 3000-3500 ms time window post-problems (i.e., during the late stage). The late stage approximates the period of problem solution. Therefore, the right ventral PFC may reflect the meta-cognitive awareness of solution-obtaining or successful insight [18]. Anderson et al. [19] adopted the compound remote association (CRA) and word fragment tasks to investigate the neural correlates of insight problem solving at different stages. These authors demonstrated that solved and unsolved problems activated the left inferior (lateral) PFC in a 6-s time course prior to responding and 4-10 s after responding. However, this area exhibited differential activations across the two problem types. Activation in the left inferior PFC in solution trials was not significantly greater than in other brain areas (e.g., anterior cingulated cortex, ACC) prior to response generation. However, the left inferior PFC exhibited significantly less activation than other brain areas after response generation. In contrast, left inferior PFC activity in non-solution trials was greater throughout the task. This psychological process may be described as solution seeking or the identification of a solution to the problems prior to insight, but it primarily reflected the accomplishment of solution seeking after insight for a solved problem. However, this process remained a solution-seeking process for an unsolved problem. Activation in the left inferior PFC reflected goal-directed cognitive control and retrieval efforts for information that was relevant to the solution. These researchers also adopted a word fragment task to reexamine the effect and exclude the confounding time-accumulated effect of neuroimaging and the highly variable response times. Participants in this experiment were shown a word fragment, such as "-a-a-a", and an associated word, such as "hockey", and were given $10 \mathrm{~s}$ to complete the fragment (the intended answer is "Canada"). The study used scans at 1 and $3 \mathrm{~s}$ to reflect pre-solution activity and scans at 7 and 9 s to reflect post-solution activity to compare the participants' activity prior to response generation. Activation in the left inferior PFC in solution trials was significantly greater than in other brain areas (e.g., ACC) prior to solution. However, the left inferior PFC exhibited insignificantly less activation than other brain areas after solution until the end of feedback. 
The unsolved problems also prominently activated the left inferior PFC. These results suggested that the inferior part of the left lateral PFC was primarily responsible for the active solution-seeking process, especially the searching and retrieving of information in long-term memory on solution.

Several researchers have also investigated the brain mechanisms of prototype-induced insight. Qiu et al. [20] adopted the many-to-many prototype heuristic paradigm to examine the neural correlates of insight. These authors demonstrated that insight events activated the left middle frontal middle gyrus and inferior frontal gyrus, but noninsight events did not activate these areas. Non-insight events only activated the medial frontal cortex. These results suggest that the left middle frontal middle gyrus and left inferior frontal gyrus play important roles in the breaking of mental set and the formation of novel associations. A one-to-one comparison of the unsolved and solved problems revealed that the PFC did not exhibit any activation differences in the two types of problems [21]. The solving of these problems involved less breaking of the mental set in the one-to-one prototype heuristic condition. Therefore, participants may use prototype heuristics more directly to solve problems. The participants also solved the target problem with the assistance of prototype riddle problems in the many-to-many condition. However, this condition included many target riddle problems in a single trial, which reduced the mental set more than set shift in the many-tomany condition because the participants should have excluded the set that was elicited by the other prototype riddle problems in the same trial. Therefore, the participants adopted a larger mental set that was elicited by the prototype problems to solve the target problems consciously or unconsciously in the one-to-one prototype heuristic condition as opposed to the many-to-many condition. The left middle frontal middle gyrus and inferior frontal gyrus may be associated with set shift and working memory. The results on the neural correlates of insight in the one-to-one condition demonstrated that successful problem solving, as opposed to the successful recognition of solutions, elicited a more negative deflection in the left inferior frontal gyrus from 300 to $350 \mathrm{~ms}$ after the onset of the target, which suggests that the ERP component is linked with semantic retrieval [22]. Previous neuroimaging studies on working memory have also revealed that the left inferior frontal gyrus is responsible for semantic retrieval [23]. Anderson et al. [19] demonstrated that the left inferior PFC primarily represents working memory. Goel and Vartanian [7] also demonstrated that the DLPFC was primarily responsible for working memory and conflict resolution. These findings suggest that the left inferior frontal gyrus is primarily involved in working memory, but the left frontal middle gyrus is primarily associated with set shift. Jung-Beeman et al. [11] also observed that solved problems exhibited more activation in the bilateral inferior frontal gyrus and left medial frontal lobe compared to unsolved problems, although the activation in the bilateral inferior frontal gyrus was not significant. The CRA task involves more novel association and less mental set than the guessing riddle task, and therefore, the activation of the left middle frontal gyrus was not significant. Other studies on insight problem solving in prototype heuristic tasks have predefined insight problems as problems that generally elicit more positivity in the time window from 1200 to $1500 \mathrm{~ms}$ after the onset of problems in the control condition. The origin of the difference wave was located in the left inferior frontal gyrus [24]. The left inferior frontal gyrus was associated with the breaking of mental set and the resolution of cognitive conflict in this context. Luo et al. [15] presented a series of routine and insight riddle problems to participants and asked them to take initiative and solve these problems prior to the presentation of the solutions. This research required participants to judge whether their generated solutions were identical to the subsequently presented solutions. Brain activation was simultaneously obtained using fMRI. Neuroimaging results revealed that "Aha!" events exhibited more activation in the anterior part of the left lateral PFC and other brain areas than "non-Aha!" events, and the difficult-to-comprehend events, but not the "non-Aha!" events, activated the bilateral PFC. There is a difference in difficulty between the "Aha" events and the difficult-to-comprehend events. Therefore, the aforementioned results suggest that the bilateral PFC is involved in the cognitive processing of problem difficulty. The difference in the cognitive processes of both events is that the key process underlying the "Aha!" events was the breaking of mental set, but the cognitive process underlying the difficult-to-comprehend events was the maintenance of mental set. Therefore, the breaking of mental set was primarily associated with the right PFC rather than left PFC because the difficult-to-comprehend events also activated the left PFC. Random effect analysis revealed that all three blocks were associated with robust left lateral PFC activity relative to the resting state. The activity in left lateral PFC did not decrease from the first block to the second and third blocks, which suggests that the left lateral PFC was not associated with problem familiarity. The aforementioned analysis revealed that the left lateral PFC was responsible for the cognitive processing of problem difficulty, and it was associated with set shift. In brief, the left PFC is primarily associated with working memory, problem difficulty and the set shift that underlie insight problem solving. If the task-related activation areas were adjacent to the superior part of the left lateral PFC, it was easier for these areas to assist the right PFC in the breaking of mental set. If the activation areas were adjacent to the inferior part of the left lateral PFC, it was easier for these areas to participate in working memory.

Several studies have adopted the number reduction task (NRT) to investigate the brain mechanism of delayed insight $[25,26]$. These experiments presented a serial of digits to participants and asked them to generate a solution to the 
problem according to the "identity rule" and the "difference rule". The responses followed a hidden rule of those digits in a trial, and this fact was importantly not mentioned to participants. The last two responses were always mirrored by the prior two responses, but the actual digit strings and responses changed from trial to trial. Insight was defined when participants finished the task with at least 36 correct shortcuts in a single block. Neuroimaging revealed that the unsolved problems exhibited more activation in the bilateral ventral medial PFC and motor areas. However, the insightful and solved problems exhibited more activation in the right dorsal medial PFC and DLPFC. These authors hypothesized that the PFC was associated with rule finding and off-line memory in the delayed insight task. The role of PFC in insight problem solving is complex, and it is associated with distributed neural networks. The PFC may be associated with conscious processing, especially conscious awareness, memory retrieval and mental imagery [25]. The time point of the occurrence of insight was difficult to identify due to the unpredictability and suddenness of insight and the coarse temporal resolution of fMRI. One study adopted high temporal resolution ERPs to capture the time course of insight occurrence in a mathematical insight problem-solving task and observed activation in the right PFC during insight [26]. The mental set in NRT was not obvious, but the participants adopted the so-called "identity rule" and "difference rule" to solve NRT problems. Therefore, the NRT included some degree of mental set. The right PFC may be involved in the cognitive processes that underlie routine strategy selection and the breaking of mental set.

The PFC is an important control and executive center, and it plays a critical role in certain higher cognition processes. Insight is a type of human thinking that naturally recruits the PFC. Many studies consistently reveal the important role of the PFC in insight problem solving using electrophysiological measures [21-28] and neuroimaging techniques [18-20,25,29]. However, the activation patterns are different across task types, research paradigms, reference states and the resolution of neuroimaging techniques. These studies consistently demonstrate that insight, as opposed to routine, events exhibit activation in the bilateral DLPFC and ventral lateral PFC, especially the right ventral lateral PFC. Functionally, the bilateral PFC may be associated with set shift in the insight task. The right lateral PFC plays a significant role in the breaking of mental set, which is independent of task types. However, the association of the left lateral PFC in insight problem solving depends on the task type, research approach and the activated areas in a task. Insight task activation of the superior part of left lateral PFC increased the probability that this brain area was associated with set shift. Activation of the inferior parts of the left lateral PFC suggested that this brain area was responsible for working memory and memory retrieval. In addition, the left lateral PFC may be involved in the cognitive processing that underlies problem difficulty and cogni- tive inhibition. The nature of activation (i.e., activation or deactivation) depended on the role that the PFC played in an insight task. The PFC is usually involved in general strategy cognition and functional modulation. The PFC would be activated if it was associated with working memory and cognitive inhibition. Individuals without a PFC may perform better in solving insight problems if the PFC does not participate in cognitive inhibition and control. One lesion study and several non-invasive brain stimulation studies have reported the aforementioned effect. Patients with focal damage to the lateral frontal cortex performed better in insight problem solving than a group of healthy participants. These results suggest that the PFC, especially the DLPFC, is primarily involved in cognitive inhibition, routine thinking and cognitive control.

\section{Cingulate cortex}

The cingulate cortex is an anatomically important part of the limbic lobe. The cingulated cortex is located in the center of the cerebrum, and it is wrapped around the central bundle of nerves known as the corpus callosum. The anterior and caudal parts of the cingulate cortex are significantly different in morphology, and this area has been divided into the anterior cingulate cortex (ACC) and posterior cingulate cortex (PCC). The cingulate cortex plays an important role in responses to monitoring, error detection, cognitive conflict, working memory and higher cognition and psychological processes that are associated with emotional arousal [30-36]. The cingulate cortex is significantly activated during insight problem solving. The PCC has been associated with insight problem solving, but most studies report an association of the ACC with insight $[11,22]$. This study reviews the role of the cingulate cortex, including the ACC and PCC, in insight problem solving.

Luo and Niki [10] recorded neural activity using fMRI and correlated activity with cognitive insight. These authors presented subjects with a series of riddle problems and general questions and asked them to actively think and discover the correct answer in a given time period. The answer was presented to the subjects after a period of time for thinking, and brain activities were scanned simultaneously. Insight events significantly activated the right anterior cingulated cortex relative to non-insight events. The non-insight events significantly activated the bilateral PCC and left ACC compared to the insight events. Events that were difficult to understand (i.e., events that subjects failed to generate a correct answer during the problem presentation phase but understood the given answer) only activated the left ACC compared to non-insight events. The difficult-to-comprehend events did not activate any cingulate regions compared to insight events. The insight task consistently activated the ACC and the left PFC compared to the resting state. Random effect analysis revealed that the activity in the ACC 
sharply decreased from the first experimental block to the second and third blocks, which suggests that the ACC is associated with the cognitive conflict between old and new ideas. The present study provides a detailed analysis of the prominent cingulate areas across various conditions. Insight events did not exhibit prominent activation in the left ACC, which contrasts the difficult-to-comprehend events. However, both the non-insight events and the difficult-to-comprehend events exhibited prominent activation in the left ACC compared to the insight events. These results suggest that the left ACC does not represent a unique mechanism of insight, but this area plays a role in a universal strategy mechanism. The activity in the left ACC was a function of problem type; the difficult-to-comprehend events elicited the greatest activation in the left ACC. The activation of the left ACC by non-insight events was less than the activation by difficult-to-comprehend events but greater than the activation by insight events. The degree of activation strength should be transitive if these events activated the same region of the left ACC. The activity in the left ACC that is elicited by the difficult-to-comprehend events should be significantly stronger than the insight events. However, no difference in left ACC activity between these two types of events is observed, which suggests that the transitive process did not exist. Some differences in the exact activation regions of the left ACC between the difficult-to-comprehend and insight events has been observed. The left ACC may be primarily responsible for cognitive monitoring or "early warning" in these events [2]. Previous studies have shown that it was difficult for meta-cognition to monitor the time course of insight. The activity in the left ACC that was elicited by non-insight events was associated with the meta-cognitive monitoring of problem solving. However, the activity that was elicited by the difficult-to-comprehend events was related to meta-cognitive awareness or the early warning of the appearance of a potential mental impasse. The similar activities in the left ACC between the difficult-to-comprehend and insight events may be a product of the number of their average trails. These non-differing effects suggest that an association of the ACC to the breaking of mental set and the "Aha!" experience post-solution is not necessary. However, the insight events exhibited a prominent activation in the right ACC, which suggests an association with cognitive conflict. One explanation is that the insight events were primarily defined as the events in which the participants' solutions did not match the given answers (i.e., the participant-generated solutions were better or worse than the given solutions, but they were not identical). Non-insight events in which participant- generated solutions were consistent with the given answers exhibited significant activation in the bilateral PCC. The right ACC may be associated with the detection of cognitive conflicts between old and new ideas during insight problem solving and resolution. The specific role of the bilateral PCC is not clear, but neuroimaging studies on creative thinking have shown that the right PCC is activated in novices operating a digit count task [37]. Neuroimaging studies on the neural correlates of economic and moral decision-making demonstrate that the bilateral PCC (especially the right PCC) is associated with the expected reward probability of expected economic utility [38], expected moral utility, practical moral utility, and event probabilities (primarily right PCC) [39]. The PCC may assist some motor areas in conflict resolution after the prefrontal cortex has monitored the response conflict [40,41]. Therefore, the right PCC may be primarily responsible for risk and confidence assessment in decision-making, which suggests that the participants were more confident in judging the non-insight response. The left PCC primarily assists the right PCC in risk assessment and problem solving, especially in reaching a final decision. However, Jung-Beeman et al. [11] and Qiu et al. [22] used the CRA task and prototype heuristic paradigm, respectively, to demonstrate that insight problems dominantly activated the left PCC compared to non-insight problems. These two tasks do not involve a cognitive conflict between old and new ideas. The ACC, which participates in the detection and resolution of cognitive conflict, was not significantly activated. However, Jung-Beeman et al. [11] demonstrated that insight and noninsight problem solving activated the left PCC, and insight problem solving activated this area earlier. Activation of the left PCC was likely associated with the act of key pressing rather than problem solving because insight problem-solving activation was faster than non-insight problem solving, and the solving of both types of problems involved key pressing. However, Qiu and Zhang hypothesized that the PCC was also related to an early warning of mental impasse.

Aziz-Zadeh et al. [18] presented a series of moderately difficult word variants and asked participants to adjust non-word letters to form words (e.g., "oxima" was adjusted into "axiom"). Comparisons between insight and search solutions revealed that the ACC was activated in both conditions, but the activation level was greater in the insight condition. These authors divided the process of problem solving into three stages and primarily focused on the signal change in the question and solution phases to specify the role of ACC in insight problem solving. Insight solutions dominantly activated the ACC in the solution phase (3 to $3.5 \mathrm{~s}$ interval post anagrams) compared to non-insight solutions, which suggests that the ACC is a critical region for the successful solving of insight problems. The monitoring of insightful processes by meta-cognition is difficult, and the ACC may not be involved in the cognitive monitoring of insight solutions. The ACC may work in concert with the PFC by evaluating the need for executive control and relaying the need for control to the PFC. Therefore, the ACC is part of a tight network that is involved in focused attention, monitoring, and executive control, and it may be a necessary component of insight processing. Anderson et al. [19] adopted two similar word association tasks (CRA and word fragment tasks) to investigate the neural correlates of insight 
problem solving. The solved problems, but not the unsolved problems, activated the left ACC in the 6-s time interval prior to responding and in the $4-10 \mathrm{~s}$ after responding. The left ACC and PFC exhibited the same degree of activation prior to insight in the solved trials, but the activity of the ACC was significantly stronger than the PFC after insight. In contrast, activation of the ACC was weaker throughout in the unsolved trials. These authors adopted a word fragment task for further investigation. The researchers used scans at 1 and $3 \mathrm{~s}$ to reflect pre-solution activity and scans at 7 and 9 s to reflect post-solution activity to compare participants' activity prior to response generation. Activation in the left ACC in solution trials was significantly smaller than the PFC prior to solution. However, the left ACC exhibited insignificantly less activation than the PFC after the solution to the end of feedback processing. Activity in the left ACC was weaker than the PFC throughout. The word fragment task is commonly used in memory studies, but it is also applicable for the examination of the cognitive processes that underlie insight due to its gestalt characteristics, such as cloze. These data suggest that activation in the PFC is linked with retrieval information for the solutions from memory, but the left ACC is primarily responsible for goal directing and governing during problem solving. Naturally, the PFC and ACC exhibit different activation patterns preand post-solution.

Luo et al. [29] demonstrated alterations in ACC activation due to the familiarity of the structure of puzzles. The ACC was less activated when the structure of the puzzle was known. Event-related fMRI was used to compare the neural correlates of the solving of two kinds of puzzles. In one condition, the subjects solved a list of puzzles that were constructed using different principles. In the other condition, the puzzles were constructed using the same principle. Therefore, the solvers could allocate some general task strategies to solve the puzzles in the second condition. This top-down control was relatively difficult to achieve in the first condition. Both conditions evoked comparable activities in the left ACC relative to resting baselines, but the first condition evoked more ACC activity than the second condition. This difference was primarily exhibited in the number of voxels. This result implies that the function of the ACC in the insightful solving of puzzles was an "early warning system" when the top-down control had failed, but the ACC did not participate in the regular top-down attentive control. The left ACC reflected general top-down information processing, but the right ACC reflected early warning processing. This interpretation has been supported in subsequent studies. Qiu et al. [20] investigated the brain mechanism of insight in the condition of the "many-to-many" prototype heuristic. The prototype problems did not significantly activate the cingulate cortex, but the solving of prototype problems (insight events), but not target problems (non-insight events), dominantly activated the left ACC and the right PCC. Therefore, both the left ACC and right PCC participate in the solving of prototype problems. If prototype problem solving is the process of solution-seeking by participants based on their previous experience and knowledge, then the target problems were solved using the rules hidden in the prototype problem solving, or at least inspired by the hidden rules. These results revealed that the cingulate cortex was less activated when the participants knew the rule or strategy of problem solving. Similar results were observed in another study. Darsaud et al. [25] adopted an NRT task to investigate the neural correlates of delayed insight. Activation in the left ACC and bilateral PCC was greater at post-solution than pre-solution. This result implies that insight problem solving activates the left and right ACC regardless of whether the problem is a predefined insight problem or is solved using the insight strategy.

The cingulate cortex is important for insight problem solving. EEG studies on insight also support this view. Qiu and Zhang [22] investigated the neural correlates of prototype-induced insight in the one-to-one condition. The successful problem solving, but not the successful recognition of solutions, elicited a more negative deflection in the left PCC from 300 to $350 \mathrm{~ms}$ after the onset of the target. The PCC may have assumed a similar role as that of the ACC because the activation in the PCC was only produced by the coarse source analysis. The ERP component may be primarily responsible for the monitoring and inhibition of mental set and cognitive conflict. Mai et al. [42,43] adopted a riddle-guessing task to examine the ERP effect of "insight" that was catalyzed by the answer to the riddle. Insightful responses induced a difference ERP component that was similar to the N400 in the ACC compared to the routine responses. Qiu et al. [44,45] also observed similar results. Insightful and difficult-to- comprehend responses elicited an N400-like difference component relative to the routine responses. These results suggest that the N400-like difference ERP component did not represent the breaking of mental set, but it reflected the cognitive conflict that was induced by old and new ideas prior to and after providing answers. This explanation seems more reasonable. Unfortunately, the source analysis results of Qiu et al. [44,45] revealed that the difference component of incomprehension did not originate in the ACC, but the origin of insight difference component was located in the ACC. Qiu et al. [21] and Wang et al. [24] further studied the insight processes that are induced by successful problem solving by participants' own initiatives. Successfully solved riddles elicited a more negative difference component in the left ACC in the time course of 1500$2000 \mathrm{~ms}$ and in the PCC at 2000-2500 ms post-problems compared to unsuccessfully solved problems [21]. Predefined insight problems elicited a more negative difference component in the right ACC in the 300-800 ms time course compared to routine problems [24]. These observations suggest that the left ACC was primarily responsible for the breaking of mental set and the formation of novel association [21], but the right ACC was primarily involved in the 
detecting and monitoring of cognitive conflict as an index of cognitive control [24].

In conclusion, considerable research using different insight tasks has been conducted on the cognitive processes underlying insight problem solving. Inconsistent operational definitions of insight, different methodologies and techniques and various research approaches have been used in previous studies on the neural correlates of insight. However, the data consistently exhibited an important role for the cingulate cortex in insight problem solving. Gaps in the specific roles of the cingulate cortex exist, but the present study illustrates the functions of the left and right cingulate cortex, especially the cognitive function of the ACC in insight problem solving. The left ACC is primarily involved in task-related goal directing and governing and the early warning of potential mental impasse. The right ACC primarily participates in the detection and resolution of the cognitive conflict that is induced by old and new ideas. The cognitive functions of the bilateral PCC exhibit some differences between various insight tasks. Most studies suggest that the right PCC is associated with the assessment of the comprehensive progress of problems. The left PCC might assist the right $\mathrm{PCC}$ and/or cognitive system (primarily the PFC) in the measurement of multiple choices and the final response. Other studies suggest that the cognitive function of the PCC is similar to the ACC, and these areas play the same role in insight problem solving. However, this hypothesis has not been validated.

\section{Hippocampus, superior temporal gyrus and fusiform gyrus}

The hippocampus, superior temporal gyrus (STG) and fusiform gyrus are typical activation regions that are often observed in insight studies. Numerous studies have observed activation in the medial temporal lobe, especially the hippocampus, parahippocampus, STG and fusiform gyrus, during insight problem solving. The current study focused on the functions of the hippocampus, STG and fusiform gyrus due to their important roles in insight. The function of the hippocampus [10] and STG [11] is emphasized because the first two studies on the neural basis of insight explored the roles of these areas in insight problem solving.

The hippocampus is a major component of the limbic system, and it is located in the medial temporal lobe. The hippocampus is primarily responsible for spatial memory and navigation, but it also has other functions. The hippocampus exhibits a well-defined structure in different species. These factors contributed to the extensive study of the hippocampus (A journal is named Hippocampus). The hippocampus has been investigated in animal studies and human studies. Animal studies have primarily examined the impact of hippocampal lesions on exploratory behaviors and the activation states of hippocampal neurons during the search for a road. Human studies have explored the relationship between the hippocampus and episodic and declarative memory [2]. Redish [46] argued that the function of hippocampus in animals and humans is similar. The hippocampus functions as a constant corrector of the errors that occurs in self-oriented behaviors, episodic recall and the filling of vacancies in a scene $[2,10]$. These functions are typically reflected in re-directed animal behaviors. If a path is obvious and clear, an animal may recover from the loss of the path despite hippocampal damage. However, hippocampus-damaged animals find it difficult to identify a path when the road is not obvious. Animals can only fulfill selforientation successfully if their actions and external information are integrated effectively [2]. A key feature of insight is the re-orientation of thinking or the transformation of problem representation. Therefore, the hippocampus may participate in insight problem solving. This hypothesis has been validated in numerous brain imaging and ERP studies on insight.

Luo and Niki [10] performed the first study on the neural mechanisms of insight. These authors applied event-related fMRI to record the brain activity of 7 college subjects during the insightful solving of riddle problems. Insight events markedly activated many distributed brain regions relative to the resting state, but the greatest activation was located in the right hippocampus. Neuropsychological studies on human hippocampus damage (e.g., H. M. patient) and previous neuroimaging studies on normal subjects demonstrated that the hippocampus is activated by novel stimuli. However, the stimuli and objects that were used in these studies (e.g., words and characters) are well-known and common in daily life, such as "mosquito" [2], which suggests that the activity of the hippocampus was not due to the novelty of the stimulus itself. Activity of the hippocampus may be induced by the (novel) associations between riddle problems and their answers. This hypothesis has been supported by many neuroimaging and ERP/EEG studies. Jung-Beeman et al. [11] adopted a CRA task based on connection theory to capture the brain mechanisms underlying the formation of remote association. The bilateral hippocampus was activated at the moment of insight, but this activation was not significant. More compelling evidence comes from Darsaud et al. [25] who adopted fMRI to scan the brain activity of repeated attempts and rule finding prior to and after insight. The left hippocampus was markedly activated in successful solvers compared to unsuccessful solvers. The connections between the hippocampus and parahippocampus in successful solvers were significantly stronger than in the unsuccessful solvers. These observations revealed that the significant activation in the left hippocampus was associated with rule finding. However, the activation of the left hippocampus gradually weakened after insight and the discovery of the hidden rule, but the connection between the hippocampus and surrounding areas was markedly enhanced. These results suggest that the hippocampus reflects the cog- 
nitive processes underlying the forming of novel association, and the activity is enhanced by the neural connections and associations with surrounding neural structures. These results fully explain the association of insight with the hippocampus [10] in some studies, but others have observed associations with the parahipp-ocampus [22,24] or temporal regions that are adjacent to the hippocampus and the parahippocampus gyrus (e.g., STG [11,15] and temporoparietal junction $[21,25,29])$.

STG is an important temporal area for insight problem solving $[11,27,45,47,48]$. The STG is located in the superior part of the lateral temporal lobe. Jung-Beeman et al. [11] first discussed the role of the STG in insight problem solving. These authors adopted the CRA task (e.g., boot, summer, ground; solutions: camp) to examine the neural correlates of insight problem solving. fMRI revealed an increased signal in the right anterior STG for insight, but not non-insight, solutions, and scalp EEG recordings exhibited a sudden burst of high-frequency (gamma-band) neural activity in the same region just prior to insight (approximately $0.3 \mathrm{~s}$ ), but not non-insight, solutions. Previous studies on speech comprehension have demonstrated that the STG plays an important role in remote semantic integration. Therefore, the STG may represent the formation of the novel remote semantic associations that underlie insight problem solving. Luo et al. [10,15,16,29] also observed activation in the right STG or its neighboring brain regions in the riddle guessing task and the chunk decomposition of Chinese characters task. The process that the subjects used to solve a list of puzzles that were constructed by different principles significantly activated the right STG (mainly BA 38) relative to the resting state, but the process of solving puzzles that were constructed using the same principle did not activate the STG or nearby areas relative to the resting state [29]. LCD, but not TCD, problems significantly activated the right STG (BA 22) during problem solving. The right STG was negatively activated across the two problem types. The activation in the right STG was negative in both the LCD and the TCD conditions, but it was more negative in the LCD condition. TCD problems are considered insight problems, and the LCD problems are considered routine problems [16]. Routine (LCD) problem solving exhibited more negative activation in the right STG, which suggests that insight (TCD) problem solving exhibited less activation in this region. These results verified the outcomes reported by Jung-Beeman et al. [11] in the opposite direction, which suggests that TCD problem solving is associated with more novel and remote associations. Many factors can explain the negative activation in the right STG. One factor addresses whether the insight problem was solved using the participants' own initiatives or passive thinking. The activation in the right STG was negative when the insight problem was solved with the assistance of a trigger (either a hint or heuristic) because the participants only partially experienced or comprehended the novel or remote association (similar to outsight) rather than forming and constructing the entire novel or remote association using their own initiative. This hypothesis was confirmed by Qiu et al. [20] who observed that prototype problem solving exhibited greater activation in the bilateral STG under the "many-to-many" prototype heuristic condition compared to target problem solving. However, the prominent activation in these regions did not occur in reverse comparisons. The prototype problem was solved by participants' own initiatives, but the target problem was solved with the assistance of the heuristic that was induced by the prototype problem. The two types of problems were very similar in structure and other aspects, but the subjects' initiatives were different. Therefore, the STG was associated with the formation of novel or remote associations using initiatives.

The role of the temporal lobe in insight problem solving has been revealed by studies of the hippocampus and STG and other brain regions, such as the fusiform gyrus $[19,20$, 49], temporal pole [18] and temporoparietal junction (TPJ) $[25,47,50]$, which includes the superior temporal sulcus and inferior parietal lobe. The hippocampus is the area between the collateral fissure and the hippocampal fissure. The parahippocampus is the hook-like part that surrounds the anterior part of the hippocampal fissure. The fusiform gyrus is adjacent to the hippocampus and parahippocampus in the area between the inferior temporal sulcus and collateral fissure. These data suggest vigorous information communication and neural connections between the hippocampus and parahippocam-pus gyrus due to the anatomical proximity. Numerous studies have demonstrated that the activation in the fusiform gyrus $[19,20,49]$ is associated with some insight tasks. Qiu et al. [20,49] successively adopted fMRI and ERPs to investigate the neural mechanism of insight problem solving. Their study revealed that prototype problem solving significantly activated the left fusiform gyrus in a prototype heuristic task, but target problem solving did not activate this brain region. A single character rearrangement task also demonstrated marked activation in the same region relative to the control condition. However, Aziz-Zadeh et al. [18] did not observe significant activation in this brain area in an fMRI study using a similar English single-word rearrangement task. The activity in the left fusiform gyrus was primarily associated with the breaking of mental set and the restructuring of Chinese characters chunks [49]. The present study argues that the fusiform gyrus participates in the formation of novel and remote associations as a candidate of the hippocampus and STG. The following three reasons support this hypothesis. First, the fusiform gyrus is an important visual word form area (WFA) [51]. The activity of this region may be related to the visual processing of word forms because Zhang et al. [49] and Qiu et al. [20] demonstrated some activation of the fusiform gyrus during the visual processing of word forms. The different results of the activation in the fusiform gyrus may be explained by differences in experimental materials, but the neuroimaging 
study of Aziz-Zadeh et al. [18] and other ERP/EEG studies $[49,52]$ do not reveal activation in the same brain area. This difference is likely because Chinese characters (especially the Chinese pictophonetic characters) exhibit more visual features and prominence during processing compared to English words. However, Luo et al. [16] did not observe marked activation in the fusiform gyrus using a Chinese characters chunk decomposition task. Second, the fusiform gyrus is an important brain area that is responsible for face recognition [53,54]. This area is also involved in the theory of mind and intention inference [55-58]. The fusiform gyrus may exhibit a function similar to the STG in the theory of mind [55,59], and it is primarily responsible for the identification and understanding of an actor's intention on the basis of (potential) social clues. The difference is that the clues that are adopted by the STG to fulfill the above function are primarily derived from auditory and sound stimuli, but the clues that are adopted by the fusiform gyrus are primarily derived from facial and other visual stimuli (e.g., pictures). Clue identification and intention understanding require the integration of large amounts of information and the connections between this information. Additionally, individuals mainly visually process problems at the moment of insight [2]. Thus, the fusiform gyrus may be involved in the formation of novel and remote associations. Finally, the hippocampus may not form novel and remote associations in isolation, but it requires cooperation from surrounding neural structures and connections. The fusiform gyrus is a key region near the hippocampus and STG, and it was naturally involved in the formation of novel associations. The distributed temporal regions and other brain areas, such as the temporoparietal junction, constitute a neural network that specializes in the formation of novel and remote associations.

In summary, existing research has observed a predominant activation in the hippocampus, STG and fusiform gyrus at the moment of insight. The temporal lobe is the critical neural basis for the cognitive processes that underlie creativity and creative expression. For example, a threefactor anatomical model suggests that the temporal lobe is primarily responsible for the formation of novel connections and the enhancement of connection quality [14,60,61]. Numerous studies have demonstrated that the hippocampus, STG and fusiform gyrus participate in the formation of novel connections and remote associations. The hippocampus, STG and fusiform gyrus may form a neural network that is responsible for the formation of novel and remote associations in insight problem solving. The hippocampus, STG and fusiform gyrus are responsible for the formation and construction of novel and remote associations of different types. The hippocampus is mainly responsible for the formation of novel associations based on previous experience and the knowledge that is in memory. The STG is primarily involved in the formation of remote associations and novel connections from auditory and semantic elements.
The fusiform gyrus primarily participates in the formation of novel images and remote associations based on visual imagery. These areas form an integrated network that specializes in novel connections and remote associations. However, these areas may also participate in other types of association and assist other nodes in the integrated network to generate and form novel associations through rich neural connections. More importantly, these areas generate and construct more complex and rich novel images and associations.

\section{Precuneus and cuneus}

Precuneus and cuneus are two critical brain regions in insight. Luo et al. [29] investigated the brain mechanisms that are involved in insight using a riddle guessing task and compared brain activations that corresponded to the solving of two kinds of puzzles. The solving of brainteaser puzzles that were constructed using different principles was associated with activation in the ACC, the left precuneus and other frontal brain areas compared to the resting baseline. The solving of "homophone" puzzles that were constructed using the same principle was associated with activities in the bilateral inferior frontal gyrus and the left precuneus compared to the resting baseline. No differences in the activation of the precuneus were noted. A neuroimaging study [2] on the brain mechanisms of brainteasers and encyclopedic knowledge problem solving revealed that the solving of the former problems exhibited more activation in the left precuneus compared to the latter problems, but the latter problems exhibited more activation in the bilateral cuneus compared to the former problems. Neuroimaging demonstrated that [20] the solving of target riddles significantly activated the left precuneus compared to the solving of prototype riddles. However, Luo and Niki [10] did not obtain similar results. These authors demonstrated that when the answers were presented to subjects, the solutions to brainteasers, which require the breaking of mental set and the transformation of conventional thinking, significantly activated the right, but not the left, precuneus. Luo et al. [16] further investigated the roles of the cuneus and precuneus in insight problem solving using a novel chunk decomposition task. Chunk decomposition of TCD problems significantly and negatively activated the right cuneus compared to LCD problems. The TCD problems uniquely and negatively activated the left and right cuneus compared to the LCD problems. Luo et al. suggested a constructive framework for interpretation [2] and proposed that the precuneus played a general strategy role in insight and routine problem solving. The precuneus may also work in conjunction with the cuneus, bilateral posterior middle temporal gyrus, and middle occipital gyrus. These brain regions formed a "non-verbal" visuospatial information processing network that associated with the effective transformation of problem representation. This hypothesis has been supported by numerous studies, 
and it is typically exhibited in the following three aspects. First, the precuneus (including some areas of cuneus) is primarily involved in retrieval from working memory or episodic memory, especially the retrieval and processing of spatial images [62-65]. Second, numerous great scientific discoveries and inventions throughout human history have been non-verbal. For example, Einstein said "These thoughts did not come in any verbal formulation. I rarely think in words at all. A thought comes, and I may try to express it in words afterward". Finally, many traditional behavioral studies on protocol analysis and the feeling of knowing (FOK) in cognitive psychology also support the above hypothesis [2]. FOK (e.g., feeling of warmth) cannot predict the time of the appearance of insight or "Aha!" in insight problem solving, but it accurately foresees the progress of routine problem solving. Some laboratory studies have also demonstrated that solvers usually remain silent for seconds or minutes before the flash of insight [2]. These studies indicate that the insight may appear in a visual rather than auditory form.

Darsaud et al. [25] adopted the NRT to investigate the brain mechanisms of rule finding. The entire course was divided into two stages according to the time point of the successful discovery of the hidden rule. The cognitive process in the post-phase of rule finding exhibited more activation in the bilateral cuneus compared to the pre-phase (primarily the seeking of the hidden rule). The process after a successful insight, (i.e., participants successfully identified the hidden rule and applied it to solve problems) significantly activates the right precuneus relative to the solution-seeking process before insight. These results are similar to those of Oishi et al. [66] who examined the brain mechanisms that are associated with the reduction in reaction time (RT) in serial reaction time tasks (SRTTs). Participants were instructed to press one of four buttons that corresponded to visual stimuli as quickly as possible and with minimal errors during fMRI. Stimuli were presented either in random order (control condition) or in a repeated 6-item sequence (learning condition). Subjects with high RT reduction exhibited more prominent activation in the bilateral precuneus than subjects with low RT reduction. Intra-subject correlation analysis revealed that time course of precuneus activation was unrelated to the time course of RT reduction. However, inter-subject correlation analysis revealed that RT changes only correlated with precuneus activation, which suggests that subjects with more prominent RT reduction exhibited more prominent activation of the bilateral precuneus. The results illustrate that the bilateral precuneus played a critical role in the control of finger movements with reference to buffered memory $[66,67]$. The moment of insight or the successful finding of the implicit rule was determined by the sharp reduction in RT when the participants fulfilled the subsequent experimental task [25]. Therefore, the precuneus may participate in visual information processing during the search and application of the hidden rule (i.e., the mirror rule) prior to and/or after the moment of insight. The precuneus may be involved in the formation and construction of a "non-verbal" visuospatial information- processing network in conjunction with the cuneus and other brain regions. However, the activation in the precuneus may also represent the rapid reduction in behavioral RT and its cortical control of finger movement.

The above two alternative explanations of the roles of precuneus and cuneus in insight problem solving are supported by their anatomical locations. The precuneus is critically linked with the parietal lobe and cerebellum, and its neural efficacy is primarily determined by its anatomical connections and nerve conduction. The activation of areas of the precuneus or cuneus that are near and connected to the parietal lobe or the parietal regions (e.g., the left inferior parietal lobule) is primarily involved in visuospatial information processing and working memory retrieval. The activation of this part of the precuneus or cuneus is likely involved in the construction and formation of "non-verbal" visuospatial information processing network. The precuneus and the cuneus may not function in isolation, but these areas may work in concert with other visual areas that have been observed in previous studies, such as the inferior occipital gyrus and middle occipital gyrus [2,20,47,68]. However, the activation of areas of the precuneus or cuneus that are near the parietal lobe and connected to the cerebellum, which regulates motor control and movement, may assist in movement control, especially the cortical control of finger movement. Therefore, the specific roles of the precuneus and cuneus require further investigation. However, these areas surely participate in insight problem solving. Appropriate portions of the precuneus and cuneus should be investigated to accurately investigate the specific roles of different regions of the precuneus and cuneus (e.g., the region near the cerebellum or adjacent to the parietal lobe) in insight problem solving.

\section{Insula and cerebellum}

The insula is a cortical part of the human brain with widespread neural connections to the frontal cortex, temporal cortex and parietal cortex. However, the insula is very difficult to observe from a lateral view because of its anatomical position. The insula is the specific brain region that is responsible for disgust [69,70]. A meta-analysis of neuroimaging results on emotional processing revealed that the insula is also associated with threat-related emotions, such as fright [71,72]. Wager and Barrett [73] provided a detailed analysis on the role of different insular areas in cognitive process. The insula is engaged in the psychological processes that underlie emotional experience, working memory and attention shift. The role of the insula in insight problem solving may be associated with the "Aha!" experience and the interactions between cognition and emotion in insight 
problem solving.

Luo et al. [15] observed that insight riddles activated the left insula compared to non-insight riddles in a Chinese riddles guessing task. However, reverse comparisons failed to reveal activation in the same region. These results suggest that the left insula is specific for insight problem solving. Darsaud et al. [25] adopted the NRT to examine the brain activation that is engaged in rule finding and demonstrated that rule finding significantly activated the left insula. A real-time analysis of the entire time course of problem solving revealed that the post-phase of rule finding activated the posterior areas of the bilateral insula compared to the pre-phase. Wager and Barrett [72] demonstrated that the anterior insula was primarily responsible for attention shifts and working memory, the upper insula near the precentral gyrus primarily represented the emotional experience, and the posterior insular regions were primarily involved in the processing of somatosensory signals. These results suggest that the insula participates in the "Aha!" experience during insight problem solving. Aziz-Zadeh et al. [18] questioned this inference with the "feeling of warmth" effect of insight problem solving in the psychological literature. A significant positive correlation between problem solving progress and the feeling of warmth during routine problem solving was observed. However, no similar correlations were noted during insight problem solving. In particular, when participants were consistently asked to describe how close they were to finding a solution for a given problem throughout the problem-solving time period, the effect of their reports on the two types of problem solving was different. For routine problem solving, participants commonly reported increasing "warmth" as they were getting closer to the solution, and pieces of the solution became more apparent to the subject. In contrast, participants commonly reported a lack of warmth until the solution was suddenly reached as a whole, seemingly out of nowhere for insight problem solving. These inconsistent results suggest that the roles of the insula in insight problem solving require further examination. Aziz-Zadeh et al. divided the entire process of problem solving into three phases. The early stage was the interval from the presentation of the target stimulus to the subsequent $2.5 \mathrm{~s}$, and the last stage was the 3 to $5.5 \mathrm{~s}$ post-target stimulus interval. A functional dissociation of insula regions across the two types of problem solving was observed; the right and left insula were activated for insight problem solving, but the left insula was uniquely activated in routine problem solving. The bilateral insulae were activated during insight solutions compared to resting, but the left insula did not exhibit significant activation in the comparison of routine problem solving with resting. These results imply that routine problem solving is primarily marked by the activation of the left insula, but insight problem solving is marked by the activation of bilateral insulae. The left insula may be involved in serial processing, and the right insula may be associated with gestalt processing because activation in the insula primarily occurred during the early stage. Different studies on insight have not reached a consensus on the function of insula in insight problem solving, but the right insula is important and necessary for insight problem solving. The present results support the view that creative insight reflects creativity, and studies commonly suggest that the right brain dominance theory in creative thinking is applicable for insight problem solving [52]. Luo et al. [16] observed that LCD problem solving significantly exhibited more activation in the right insula than TCD problem solving. The activations in both conditions were negative. Consequently, these results suggest that the right insula exhibits more negative activation during loose chunk decomposition problem solving. The negative activation of the right insula may be determined by the nature of initiative during problem solving. Specifically, LCD problems are mainly solved by participants' own initiatives, but TCD problems are primarily solved by the subsequently presented hints. The widespread connections between the insula and other cerebral regions and its unique location adjacent to the hippocampus and STG suggest that the insula may be responsible for spontaneous divergent thinking and cognitive flexibility during insight problem solving. The right insula would exhibit enhanced (negative) activation when the insight problem was solved using the subjects' own thinking. The insula may also be linked with the "Aha!" experience [74], but it requires further investigation.

The cerebellum plays an indispensable role in insight. The cerebellum is a separate structure that is attached to the bottom of the brain beneath the cerebral hemispheres and behind the portion of the brainstem called the pons. The cerebellum plays a necessary and critical role in movement-related functions, but it also participates in some cognitive functions, such as attention and language, and the regulation of fear and pleasure responses. Some types of insight problems also activate the cerebellum. Goel and Vartanian adopted a traditional insight task (i.e., Matchstick Problems) to capture the brain activity that is associated with mathematical insight problems [7]. Insight problems activated the cerebellum, and this activation covaried with the number of solutions in Match Problems. The cerebellum was thought to be associated with the cortical control of finger movement because the acquisition of the solution required key pressing. Tian et al. [75] and Qiu et al. [20] adopted event-related fMRI to investigate brain mechanisms of mental preparation and prototype-induced insight, respectively. Target problem solving exhibited more activation in the cerebellum than prototype problem solving. However, the activation in the cerebellum was not specific for insight problem solving because the cerebellum exhibited different activations during different periods of problem solving. The cerebellum may be responsible for semantic storage and memory retrieval of the knowledge of prototype riddles during target problem solving [20,75]. Participants in this study were not required to press keys during the 
study stage, but key presses were required to report the solutions. The activation in the cerebellum may be associated with this response and may primarily reflect the cortical control of finger movement.

\section{Conclusion and future prospects}

Behavioral studies on insight problem solving have demonstrated that complex problem-solving behavior is a cognitive process based on the novel thinking and grasping of the entire problem situation or space, and insight is an "emergent" rather than a "gradual" process. Cognitive neuroscience studies have revealed that insight is a function of the human brain. Insight is complicated and special because it is currently unpredictable for meta-cognition, and it occurs as a sudden silence without verbal thinking in an all-or-none manner. The cognitive processes underlying insight cannot be accomplished by a single brain region. Insight is accomplished by distributed brain areas and the widespread connections between these areas. These distributed brain regions of insight form a functional network. The functional network relies on its neural or structural network $[61,76]$. Here, the structural base of the functional network of insight is the "insightful brain". This putative framework is not an independent structural entity, but it is a neural network that is constructed by the distributed brain regions and neural substances and the neural connections between the distributed brain regions. The insightful brain involves many distributed brain regions, including the lateral prefrontal cortex, cingulate cortex (primarily the ACC and PCC), hippocampus, superior temporal gyrus, fusiform gyrus, precuneus, cuneus, insula, cerebellum and some areas of the parietal cortex. The lateral prefrontal cortex is responsible for the mental set shift in insight problem solving. The cingulate cortex is involved in cognitive conflicts between new and old ideas and progress monitoring, and the hippocampus, superior temporal gyrus and fusiform gyrus form an integrated functional network that specializes in the formation of novel and effective associations. The effective transformation of problem representation depends on a non-verbal visuospatial information-processing network that comprises the precuneus and cuneus and other regions that are distributed in the parieto-occipital junction. The insula reflects cognitive flexibility and the emotional experience that is associated with insight, and the cortical control of finger movements relies on the cerebellum. The insightful brain is a complicated structural and functional system that forms a human brain functional network, or connectome, of many different structure-specific and function-related neural correlates.

Research on the insightful brain is a novel and sharply rising research area in the 21 st century. This research was in its initial stage during the past decade (2003-2012), but this field has increasingly published innovative research. Natu- rally, many worthy problems require further investigation. Some differences in the activation of areas in different studies and experimental tasks on creative insight have been observed. These inconsistencies may be related to the cognitive processes that underlie different experiment tasks (e.g., riddles and puzzles, Chinese character-generation task), the different reference states (e.g., routine problem, unsuccessful insight problem solving, no-Aha experience), or different test procedures (insight is a passive and active process) that were used in previous studies. Increased effort is required to develop and design a reference task that is more consistent with the characteristics of subtraction experimental designs. Future studies should strengthen the investigations on cognitive functions that are associated with the different brain regions that are involved in insight. Specifically, researchers can adopt a human connectome, structural equation modeling (SEM), dynamic causal modeling (DCM) and granger causality analysis to investigate the connection modes and compatibility of different brain regions that are involved in insight and further illustrate the small-world neural network of the insightful brain [77]. More investigations on the interaction of cognition with emotion are required in future studies because (creative) insight includes cognitive and affective insight. The insightful brain involves the cognitive and the emotional or affective brain as well as the neural connections between them. Additionally, researchers should further investigate the interaction of emotion and insight, especially the influence of emotions in insight problem solving $[78,79]$. Finally, a more general purpose for the study of the brain mechanisms of creative insight is to decode creative thinking. Creative thinking and creativity play increasingly important roles in our daily life and social development. Research on insight may clarify some critical but unclear concepts and theories on creative thinking [2]. The four-stage model of Wallace and the geneplore model of Finke are well-known cognitive models of creative thinking. The former model includes a preparation stage, incubation stage, illumination stage and verification stage, and the latter model includes a generation stage and exploration stage. The neural mechanisms that are correlated with these stages have not been investigated using different creative thinking tasks, but these mechanisms have been explored using different creative insight paradigms and tasks [80,81]. Previous studies on the laterality or hemispheric effect of creative thinking have demonstrated that the left hemisphere is generally associated with analytical thinking, and the right hemisphere is associated with creative thinking. Some studies on insight problem solving support a right hemispheric dominance of insight [52], but most studies exhibit the left hemispheric effect of insight [82]. Both hemispheres are involved in creative insight, and the interaction between the two hemispheres is critical. However, only the right hemisphere is uniquely responsible for creative insight. Future studies should adopt different paradigms and experimental tasks of 
creative insight to further investigate the right hemispheric dominance theory and other theories on creative insight (e.g., neural efficiency hypothesis and inter-hemispheric interaction hypothesis [14]). Research on the insightful brain is an important method for the testing of relevant theories of creative thinking, and it plays a critical role in increasing scientific inventions and boosting social development. Researchers will delineate the myth of insight using more innovative tasks and scientific paradigms in the near future.

The authors express their great thanks to Michelle V. and Suja T. for their help on language editing, also, to editor Rui An for her work on manuscript editing. This work was supported by the National Natural Science Foundation of China (30770708), National Basic Research Program of China (2010CB833904) and the Fourth High-level Personnel Training Project in Jiangsu Province.

1 Kohler W. The Mentality of Apes. London: Routledge \& Kegan Paul, 1925

2 Luo J. Neural correlates of insight (in Chinese). Acta Psychol Sin, 2004, 36: 219-234

3 Miller E K, Cohen J D. An integrative theory of prefrontal cortex function. Annu Rev Neurosci, 2001, 24: 167-202

4 Tanji J, Hoshi E. Role of the lateral prefrontal cortex in executive behavioral control. Physiol Rev, 2008, 88: 37-57

5 Shimamura A P. A neurocognitive approach to metacognitive monitoring and control. In: Dunlosky J, Bjork R A, eds. Handbook of Memory and Metacognition. New York: Psychology Press, 2008. 373-390

6 Naghavi H R, Nyberg L. Common fronto-parietal activity in attention, memory, and consciousness: Shared demands on integration? Conscious Cogn, 2005, 14: 390-425

7 Goel V, Vartanian O. Disassociating the roles of right ventral lateral and dorsal lateral prefrontal cortex in generation and maintenance of hypotheses in set-shift problems. Cereb Cortex, 2005, 15: 1170-1177

8 Miller L A, Tippett L J. Effects of focal brain lesions on visual problem-solving. Neuropsychologia, 1996, 34: 387-398

9 Schneider F, Gur R E, Alavi A, et al. Cerebral blood flow changes in limbic regions induced by unsolvable anagram tasks. Am J Psychiat, 1996, 153: 206-212

10 Luo J, Niki K. Function of hippocampus in 'insight' of problem solving. Hippocampus, 2003, 13: 316-323

11 Jung-Beeman M, Bowden E M, Haberman J, et al. Neural activity when people solve verbal problems with insight. PLoS Biol, 2004, 2: e97

12 Gilbert S J, Zamenopoulos T, Alexiou K, et al. Involvement of right dorsolateral prefrontal cortex in ill-structured design cognition: An fMRI study. Brain Res, 2010, 1312: 79-88

13 Goel V, Grafman J. The role of the right prefrontal cortex in illstructured problem solving. Cogn Neuropsychol, 2000, 17: 415-436

14 Shen W B, Liu C, Wang Y J. Neurophysiological basis of artistic creativity (in Chinese). Adv Psychol Sci, 2010, 18: 1520-1528

15 Luo J, Niki K, Philips S. Neural correlates of the 'Aha! reaction'. Neuroreport, 2004, 12: 2013-2017

16 Luo J, Niki K, Knoblich G. Perceptual contributions to problem solving: Chunk decomposition of Chinese characters. Brain Res Bull, 2006, 70: 430-443

17 Tang X C, Pang J Y, Luo J. Zeigarnik effect in Insight problem solving: Hemispheric difference in brain activities following problem solving and during hint presentation (in Chinese). Chin Sci Bull (Chin Ver), 2009, 54: 3464-3474

18 Aziz-Zadeh L, Kaplan J T, Iacoboni M. "Aha!" The neural correlates of verbal insight solutions. Hum Brain Mapp, 2009, 30: 908-916
19 Anderson J R, Anderson J F, Ferris J L, et al. Lateral inferior prefrontal cortex and anterior cingulate cortex are engaged at different stages in the solution of insight problems. Proc Natl Acad Sci USA, 2009, 106: 10799-10804

20 Qiu J, Li H, Jou J, et al. Neural correlates of the "Aha” experiences: Evidence from an fMRI study of insight problem solving. Cortex, 2010, 46: 397-403

21 Qiu J, Li H, Yang D, et al. The neural basis of insight problem solving: An event-related potential study. Brain Cogn, 2008, 68: 100-106

22 Qiu J, Zhang Q L. "Aha!” effects in a guessing Chinese logogriph task: An event-related potential study. Chin Sci Bull, 2008, 53: 384-391

23 Wagner A D, Pare-Blagoev E J, Clark J, et al. Recovering meaning: Left prefrontal cortex guides controlled semantic retrieval. Neuron, 2001, 31: 329-338

24 Wang T, Zhang Q L, Li H, et al. The time course of Chinese riddles solving: Evidence from an ERP study. Behav Brain Res, 2009, 199: 278-282

25 Darsaud A, Wagner U, Balteau E, et al. Neural precursors of delayed insight. J Cogn Neurosci, 2011, 23: 1900-1910

26 Lang S, Kanngieser N, Jaskowski P, et al. Precursors of insight in event-related brain potentials. J Cogn Neurosci, 2006, 18: 2152-2166

27 Sandkühler S, Bhattacharya J. Deconstructing insight: EEG correlates of insightful problem solving. PLoS One, 2008, 3: e1459

28 Sheth B R, Sandkühler S, Bhattacharya J. Posterior beta and anterior gamma oscillations predict cognitive insight. J Cogn Neurosci, 2008, 21: 1269-1279

29 Luo J, Niki K, Philips S. The function of the anterior cingulate cortex (ACC) in the insightful solving of puzzles: The ACC is activated less when the structure of the puzzle is known. J Psychol Chin Soc, 2004, 5: 195-213

30 Garavan H, Ross T J, Murphy K, et al. Dissociable executive functions in the dynamic control of behaviour: Inhibition, error detection and correction. NeuroImage, 2002, 17: 1820-1829

31 Kerns J G, Cohen J D, MacDonald A W III, et al. Anterior cingulate conflict monitoring and adjustments in control. Science, 2004, 303: 1023-1026

32 Phan K L, Taylor S F, Welsh R C, et al. Activation of the medial prefrontal cortex and extended amygdala by individual ratings of emotional arousal: A functional magnetic resonance imaging study. Biol Psychiat, 2003, 53: 211-215

33 Botvinick M M, Cohen J D, Carter C S. Conflict monitoring and anterior cingulate cortex: An update. Trends Cogn Sci, 2004, 8: 539546

34 Clark L, Cools R, Robbins T W. The neuropsychology of ventral prefrontal cortex: Decision making and reversal learning. Brain Cogn, 2004, 55: 41-53

35 Davis K D, Taylor K S, Hutchison W D, et al. Human anterior cingulate cortex neurons encode cognitive and emotional demands. J Neurosci, 2005, 25: 8402-8406

36 Wang C, Ulbert I, Schomer D L, et al. Responses of human anterior cingulate cortex microdomains to error detection, conflict monitoring, stimulus-response mapping, familiarity, and orienting. J Neurosci, 2005, 25: 604-613

37 Kowatari Y, Lee S, Yamamura H, et al. Neural networks involved in artistic creativity. Hum Brain Mapp, 2009, 30: 1678-1690

38 Knutson B, Taylor J, Kaufman M, et al. Distributed neural representation of expected value. J Neurosci, 2005, 25: 4806-4812

39 Shenhav A S, Greene J D. Moral judgments recruit domain-general valuation mechanisms to integrate representations of probability and magnitude. Neuron, 2010, 67: 667-677

40 Swick D, Turken A U. Dissociation between conflict detection and error monitoring in the human anterior cingulate cortex. Proc Natl Acad Sci USA, 2002, 99: 16354-16359

41 Egner T, Etkin A, Gale S, et al. Dissociable neural systems resolve conflict from emotional versus non-emotional distracters. Cereb Cortex, 2008, 18: 1475-1484

42 Mai X Q, Luo J, Wu J H, et al. “Aha!” effects in guessing riddle task: An ERP study. Hum Brain Mapp, 2004, 22: 261-270 
43 Mai X Q, Luo J, Wu J H, et al. The ERP efffects of insight in a riddle guessing task (in Chinese). Acta Psychol Sin, 2005, 37: 19-25

44 Qiu J, Luo Y J, Wu Z Z, et al. A further study of the ERP effects 'insight' in a riddle guessing task (in Chinese). Acta Psychol Sin, 2006, 38: 507-514

45 Qiu J, Zhang Q L, Li H, et al. The ERP effects of cognitive conflict in a Chinese character-generation task. Neuroreport, 2007, 18: 881886

46 Redish A D. The hippocampal debate: Are we asking the right questions? Behav Brain Res, 2001, 127: 81-98

47 Bechtereva N P, Korotkov A D, Pakhomov S V, et al. PET study of brain maintenance of verbal creative activity. Int J Psychophysiol, 2004, 53: 11-20

48 Kounios J, Frymiare J L, Bowden E M, et al. The prepared mind: Neural activity prior to problem presentation predicts solution by sudden insight. Psychol Sci, 2006, 17: 882-890

49 Zhang M, Tian F, Wu X, et al. Neural correlates of "Aha!" effects in solving Chinese verbal problems: An event related-potential study. Brain Res Bull, 2011, 84: 210-214

50 Starchenko M G, Bechtereva N P, Pakhomov S V, et al. Study of the brain organization of creative thinking. Hum Physiol, 2003, 29: 652653

51 Cohen L, Dehaene S. Specialization within the ventral stream: The case for the visual word form area. NeuroImage, 2004, 22: 466-476

52 Shen W B, Liu C, Zhang X J, et al. The time course and hemispheric effect of "insight" in three-character Chinese riddles task: An ERP study (in Chinese). Acta Psychol Sin, 2011, 43: 229-240

53 Dolan R J, Fink G R, Rolls E, et al. How the brain learns to see objects and faces in an impoverished context. Nature, 1997, 389: 596599

54 McCarthy G, Puce A, Gore J C, et al. Face-specific processing in the human fusiform gyrus. J Cogn Neurosci, 1997, 9: 605-610

55 Schultz J, Imamizu H, Kawato M, et al. Activation of the human superior temporal gyrus during observation of goal attribution by intentional objects. J Cogn Neurosci, 2004, 16: 1695-1705

56 Barnea-Goraly N, Kwon H, Menon V, et al. White matter structure in autism: Preliminary evidence from diffusion tensor imaging. Biol Psychiatry, 2004, 55: 323-329

57 Saxe R, Kanwisher N. People thinking about thinking people: The role of the temporo-parietal junction in "theory of mind". NeuroImage, 2003, 19: 1835-1842

58 Gobbini M I, Koralek A C, Bryan R E, et al. Two takes on the social brain: A comparison of theory of mind tasks. J Cogn Neurosci, 2007, 19: $1803-1814$

59 Frith C D, Frith U. Interacting minds-A biological basis. Science, 1999, 286: 1692-1695

60 Flaherty A W. Frontotemporal and dopaminergic control of idea generation and creative drive. J Comp Neurol, 2005, 493: 147-153

61 Shen W B, Liu C, Chen J J. Neural basis of creativity: Evidence from structural and functional imaging (in Chinese). Adv Psychol Sci, 2010, 18: 1420-1429

62 Krause C M, Åström T, Karrasch, M, et al. Cortical activation related to auditory semantic matching of concrete vs. abstract words. Clin Neurophysiol, 1999, 110: 1371-1377

63 Korsnes M S, Wright A A, Gabrieli J D E. An fMRI analysis of object priming and workload in the precuneus complex. Neuropsy- chologia, 2008, 46: 1454-1462

64 Lundstrom B N, Peterson K M, Anderson J, et al. Isolating the retrieval of imagined pictures during episodic memory: Activation of the left precuneus and the left inferior frontal cortex. NeuroImage, 2003, 27: 1934-1943

65 Cavanna A E, Trimble M R. The precuneus: A review of its functional anatomy and behavioural correlates. Brain, 2006, 129: 564-583

66 Oishi K, Toma K, Bagarinao E T, et al. Activation of the precuneus is related to reduced reaction time in serial reaction time tasks. Neurosci Res, 2005, 52: 37-45

67 Luo J, Niki K, Ding Z G, et al. Precuneus contributes to attentive control of finger movement. Acta Pharmacol Sin, 2004, 25: 637-643

68 Wu Q Y, Wu L L, Luo J. What and where-the effective connectivity of dorsal and ventral visual pathways in chunk decomposition. Sci China Life Sci, 2010, 53: 1474-1482

69 Jabbi M, Bastiaansen J, Keysers C. A common anterior insula representation of disgust observation, experience and imagination shows divergent functional connectivity pathways. PLoS One, 2008, 3: e2939

70 Murphy F C, Nimmo-Smith I, Lawrence A D. Functional neuroanatomy of emotions: A meta-analysis. Cogn Affect Behav Neurosci, 2003, 3: 207-233

71 Phan K L, Wager T, Taylor S F, et al. Functional neuroanatomy of emotion: A meta-analysis of emotion activation studies in PET and fMRI. NeuroImage, 2002, 16: 331-348

72 Lane R D, Reiman E M, Ahern G L, et al. Neuroanatomical correlates of happiness, sadness, and disgust. Am J Psychiat, 1997, 154: 926-933

73 Wager T D, Feldman B L. From affect to control: Functional specialization of the insula in motivation and regulation. Published online at PsycExtra (http://www.columbia.edu/cu/psychology/tor/), 2004

74 McCrea S M. Intuition, insight and the right hemisphere: Emergence of higher sociocognitive functions. Psychol Res Behav Manag, 2010, 3: 1-39

75 Tian F, Tu S, Lv J Y, et al. Neural correlates of mental preparation for successful insight problem solving. Behav Brain Res, 2011, 216: 626-630

76 Liang X, Wang J H, He Y. Human connectome: Structural and functional brain networks (in Chinese). Chin Sci Bull (Chin Ver), 2010, 55: $1565-1583$

77 Schilling M A. A "small-world" network model of cognitive insight. Creativity Res J, 2005, 17: 131-154

78 Subramaniam K, Kounios J, Parrish T B, et al. A brain mechanism for facilitation of insight by positive affect. J Cogn Neurosci, 2009, 21: 415-432

79 Sakaki M, Niki K. Effects of the brief viewing of emotional stimuli on understanding of insight solutions. Cogn Affect Behav Neurosci, 2011, 11: 526-540

80 Wu L, Knoblich G, Luo J. The role chunk tightness and chunk familiarity in problem solving: Evidence from ERPs and fMRI. Hum Brain Mapp, 2012, doi:10.1002/hbm.21501

81 Ludmer R, Dudai Y, Rubin D. Uncovering camouflage: Amygdala activation predicts long-term memory of induced perceptual insight. Neuron, 2011, 69: 1002-1014

82 Dietrich A, Kanso R. A review of EEG, ERP, and neuroimaging studies of creativity and insight. Psych Bull, 2010, 136: 822-848

Open Access This article is distributed under the terms of the Creative Commons Attribution License which permits any use, distribution, and reproduction in any medium, provided the original author(s) and source are credited. 